IU-MSTP/15

hep-th/yy-mm-dd

August 1996

\title{
Quantum Exchange Algebra and Locality in Liouville Theory
}

\author{
Takanori Fujiwara, ${ }^{1}$ Hiroshi Igarashi and Yoshio Takimoto \\ ${ }^{1}$ Department of Physics, Ibaraki University, Mito 310, Japan \\ Graduate School of Science and Engineering, Ibaraki University, Mito 310, Japan
}

\begin{abstract}
Exact operator solution for quantum Liouville theory is investigated based on the canonical free field. Locality, the field equation and the canonical commutation relations are examined based on the exchange algebra hidden in the theory. The exact solution proposed by Otto and Weigt is shown to be correct to all order in the cosmological constant.
\end{abstract}

PACS: 11.10.Kk, 11.25.Hf, 11.25.Pm

Keywords: Liouville theory, exchange algebra, quantum group, quantum deformation 
The investigations of quantum Liouville theory [1-6] have exposed rather rich canonical structures of the theory including the discovery of exact operator solutions [1,4-6] and underlying quantum group symmetry [7-9] as extensively studied by Gervais and his collaborators. Exact solutions not only enable us detailed analyses of the system under considerations but also are useful in examining general frameworks which are considered to be applicable to the system. It is certainly desired to gain better understanding on the exact solutions.

In this note we shall investigate the exact operator solution proposed by Otto and Weigt [6] and give a complete derivation of the formal expansion of the Liouville exponential in terms of a canonical free field by postulating (1) (pseudo-)conformal invariance, (2) locality and (3) the Liouville field equation. In ref. [6] the locality conditions were solved to third order in the cosmological constant and the general formula for the Liouville exponential was proposed. It turned out to be a kind of quantum deformation of the classical expansion [9], suggesting underlying quantum group structure [8]. We shall extend the result of ref. [6] to all order. Gervais and Schnittger [10] also gave a verification based on their algebraic scheme of chiral vertex operators [8]. Here we basically follow the approach of refs. [6, 11] and show that there exists an interesting exchange algebra leading to the quantum deformation.f

Let us start from the Liouville theory described by the action

$$
S=\frac{1}{\gamma^{2}} \int d \tau d \sigma\left(\frac{1}{2} \partial_{\mu} \varphi \partial^{\mu} \varphi-\mu^{2} \mathrm{e}^{\varphi}\right)
$$

where the coupling constant $\gamma^{2}$ is related to the Virasoro central charge by $\frac{1}{\gamma^{2}}=\frac{25-D}{48 \pi}$. Using the parametrization of ref. [3], the classical solution to the Liouville field equation is given by

$$
\varphi(\tau, \sigma)=\ln \frac{\partial_{+} A\left(x^{+}\right) \partial_{-} B\left(x^{-}\right)}{\left(1+\frac{\mu^{2}}{8} A\left(x^{+}\right) B\left(x^{-}\right)\right)^{2}},
$$

with $x^{ \pm}=\tau \pm \sigma$. We will restrict ourselves to the case where $A\left(x^{+}\right)$and $B\left(x^{-}\right)$can be chosen to satisfy the periodicity $A\left(x^{+}+2 \pi\right)=\alpha A\left(x^{+}\right)$and $B\left(x^{-}-2 \pi\right)=\alpha^{-1} B\left(x^{-}\right)$with $\alpha \neq 1$ [1, 6, 11]. They can be expressed in terms of a free field $\psi(\tau, \sigma)=\psi^{+}\left(x^{+}\right)+\psi^{-}\left(x^{-}\right)$as

$$
\begin{aligned}
& A\left(x^{+}\right)=C(P) \int_{0}^{2 \pi} d \sigma^{\prime} \mathrm{e}^{\frac{1}{4} \gamma P \epsilon\left(\sigma-\sigma^{\prime}\right)+\psi^{+}\left(\tau+\sigma^{\prime}\right)}, \\
& B\left(x^{-}\right)=C(P) \int_{0}^{2 \pi} d \sigma^{\prime \prime} \mathrm{e}^{-\frac{1}{4} \gamma P \epsilon\left(\sigma-\sigma^{\prime \prime}\right)+\psi^{-}\left(\tau-\sigma^{\prime \prime}\right)},
\end{aligned}
$$

\footnotetext{
${ }^{*}$ Exchange algebra in quantum Liouville theory has already been noted in refs. 8, 12 .
} 
where $\epsilon(\sigma)$ stands for stair-step function defined by $\epsilon(\sigma)=2 n+1$ for $2 n \pi<\sigma<2(n+1) \pi$ with $n$ being an integer and $C(P)=\left(2 \sinh \frac{\gamma P}{4}\right)^{-1}$. We expand $\psi^{ \pm}$by

$$
\psi^{ \pm}\left(x^{ \pm}\right)=\frac{\gamma}{2} Q+\frac{\gamma}{4 \pi} P x^{ \pm}+\frac{i \gamma}{\sqrt{4 \pi}} \sum_{n \neq 0} \frac{1}{n} a_{n}^{( \pm)} \mathrm{e}^{-i n x^{ \pm}} .
$$

Then the zero mode momentum $P$ is related to $\alpha$ by $\ln \alpha=\frac{1}{2} \gamma P$.

The key property of the theory is the existence of a canonical transformation between the interacting theory and the free field [1, 3, 4, 6, 11]. Furthermore the improved stress tensor for the interacting theory can be expressed in terms of the free field as

$$
T_{ \pm \pm}=\frac{2 \pi}{\gamma^{2}}\left(\left(\partial_{ \pm} \psi\right)^{2}-2 \partial_{ \pm}^{2} \psi\right)
$$

This satisfies the classical Virasoro algebra and generates pseudo-conformal transformations $x^{ \pm} \rightarrow f^{ \pm}\left(x^{ \pm}\right)$, under which the Liouville exponential $\mathrm{e}^{\lambda \varphi}$ is transformed as a primary field of conformal weight $\lambda$. The conformality of the classical solution (2) can be more transparent if it is put in the form

$$
\begin{aligned}
\mathrm{e}^{\lambda \varphi} & =\mathrm{e}^{\lambda \psi}\left(1+\frac{\mu^{2}}{8} S\right)^{-2 \lambda} \\
& =\mathrm{e}^{\lambda \psi} \sum_{m=0}^{\infty} \frac{(-1)^{m}}{m !} \frac{\Gamma(2 \lambda+m)}{\Gamma(2 \lambda)}\left(\frac{\mu^{2}}{8}\right)^{m} S^{m}
\end{aligned}
$$

where we have introduced the "screening charge" $S(\tau, \sigma)=A\left(x^{+}\right) B\left(x^{-}\right)$as in ref. [8]. The conformal weight of the Liouville exponential is carried by the free field exponential $\mathrm{e}^{\lambda \psi}$, while $S$ is transformed as conformal field of weight zero.

If there exists a quantum analogue of the canonical transformation from the interacting theory to the free field, we can define quantum theory via the free field for which the quantization is rather obvious. To establish this we must find an appropriate quantum formula for the Liouville field, and verify the canonical commutation relations and the field equation.

In quantum theory we impose the commutation relations

$$
[Q, P]=i, \quad\left[a_{n}^{(+)}, a_{m}^{(+)}\right]=\left[a_{n}^{(-)}, a_{m}^{(-)}\right]=n \delta_{n+m, 0}
$$

and introduce free field operator ordering for oscillatory modes and symmetric ordering for zero modes by : $\mathrm{e}^{a Q} f(P): \equiv \mathrm{e}^{\frac{1}{2} a Q} f(P) \mathrm{e}^{\frac{1}{2} a Q}$. We assume that the conformal symmetry remains intact and is generated by the normal-ordered stress tensor (5). 
Instead of dealing with $\varphi$ directly we will argue the Liouville exponential $\mathrm{e}^{\lambda \varphi}$ for arbitrary $\lambda$. Then the Liouville field can be defined by $\left.\varphi \equiv \frac{d}{d \lambda} \mathrm{e}^{\lambda \varphi}\right|_{\lambda=0}$. Since $\mathrm{e}^{\lambda \varphi}$ should have definite conformal weight, it can be expanded as

$$
\mathrm{e}^{\lambda \varphi(\tau, \sigma)}=: \mathrm{e}^{\lambda \eta \psi(\tau, \sigma)}: \sum_{m=0}^{\infty}\left(\frac{\mu^{2}}{8}\right)^{m} C_{m}(\varpi, \lambda)(S(\tau, \sigma))^{m},
$$

where the parameter $\eta$ is chosen so that the lhs reduces to a $(1,1)$ primary field for $\lambda=1$ and

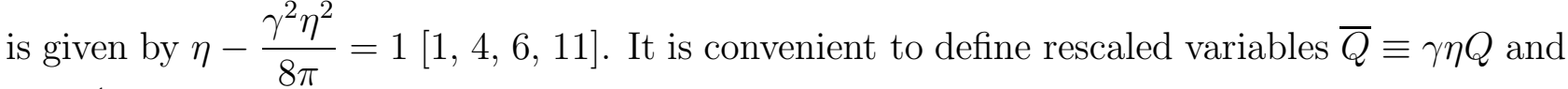
$\bar{P} \equiv \frac{1}{4} \gamma \eta P$. The screening operator $S$ must be of vanishing conformal weight and is assumed to be given by

$$
S(\tau, \sigma)=\int_{0}^{2 \pi} d \sigma^{\prime} \int_{0}^{2 \pi} d \sigma^{\prime \prime}: \mathrm{e}^{\bar{P}\left\{\epsilon\left(\sigma-\sigma^{\prime}\right)-\epsilon\left(\sigma-\sigma^{\prime \prime}\right)\right\}+\eta \psi^{+}\left(\tau+\sigma^{\prime}\right)+\eta \psi^{-}\left(\tau-\sigma^{\prime \prime}\right)}: .
$$

Since the stress tensor (5) commutes with $P$, the expansion coefficients $C_{m}(\varpi, \lambda)$ may depend on $P$ without conflicting the conformal symmetry. Here we use $\varpi$ defined by $\bar{P}=i \pi g \varpi$ with $g \equiv \frac{\gamma^{2} \eta^{2}}{8 \pi}$ for later convenience and $C_{0}(\varpi, \lambda)=1$ is assumed. The effective Planck constant $h$ used in refs. [8] corresponds to $\pi g$ in our case. The expansion (8) is formal in the sense that the operators $(S(\tau, \sigma))^{m}$ become ill-defined for fixed $g$ if $m$ is sufficiently large [6, 8, 11].

The coefficients $C_{m}(\varpi, \lambda)$ can be determined by requiring locality

$$
\left[\mathrm{e}^{\lambda \varphi(0, \sigma)}, \mathrm{e}^{\nu \varphi\left(0, \sigma^{\prime}\right)}\right]=0
$$

This is equivalent to the conditions

$$
\sum_{m+m^{\prime}=J}\left[V_{\lambda}(\sigma) C_{m}(\varpi, \lambda)(S(0, \sigma))^{m}, V_{\nu}\left(\sigma^{\prime}\right) C_{m^{\prime}}(\varpi, \nu)\left(S\left(0, \sigma^{\prime}\right)\right)^{m^{\prime}}\right]=0,
$$

where we have introduced $V_{\lambda}(\sigma)=$ : $\mathrm{e}^{\lambda \eta \psi(0, \sigma)}$ : and the summation runs over nonnegative integers $m, m^{\prime}$.

To simplify (11) we need to develop systematic way to expand the operators appearing in the lhs of (11) by a set of independent operators. To find them we follow the idea of ref. [8]. We can restrict ourselves to the case $0<\sigma^{\prime}<\sigma<2 \pi$ without loss of generality and define

$$
\begin{aligned}
A_{\sigma \sigma^{\prime}} & =\int_{0}^{\sigma^{\prime}} d \sigma^{\prime \prime} \mathrm{e}^{\frac{1}{2} \bar{Q}}: \mathrm{e}^{\bar{P}+\frac{1}{\pi} \bar{P} \sigma^{\prime \prime}+\eta \psi_{\text {osc }}^{+}\left(\sigma^{\prime \prime}\right)}:+\int_{\sigma}^{2 \pi} d \sigma^{\prime \prime} \mathrm{e}^{\frac{1}{2} \bar{Q}}: \mathrm{e}^{-\bar{P}+\frac{1}{\pi} \bar{P} \sigma^{\prime \prime}+\eta \psi_{\text {osc }}^{+}\left(\sigma^{\prime \prime}\right)}: \\
A_{\Delta} & =\int_{\sigma^{\prime}}^{\sigma} d \sigma^{\prime \prime} \mathrm{e}^{\frac{1}{2} \bar{Q}}: \mathrm{e}^{\bar{P}+\frac{1}{\pi} \bar{P} \sigma^{\prime \prime}+\eta \psi_{\text {osc }}^{+}\left(\sigma^{\prime \prime}\right)}: \\
B_{\sigma \sigma^{\prime}} & =\int_{0}^{\sigma^{\prime}} d \sigma^{\prime \prime}: \mathrm{e}^{-\bar{P}-\frac{1}{\pi} \bar{P} \sigma^{\prime \prime}+\eta \psi_{\text {osc }}^{-}\left(-\sigma^{\prime \prime}\right)}: \mathrm{e}^{\frac{1}{2} \bar{Q}}+\int_{\sigma}^{2 \pi} d \sigma^{\prime \prime}: \mathrm{e}^{\bar{P}-\frac{1}{\pi} \bar{P} \sigma^{\prime \prime}+\eta \psi_{\text {osc }}^{-}\left(-\sigma^{\prime \prime}\right)}: \mathrm{e}^{\frac{1}{2} \bar{Q}} \\
B_{\Delta} & =\int_{\sigma^{\prime}}^{\sigma} d \sigma^{\prime \prime}: \mathrm{e}^{-\bar{P}-\frac{1}{\pi} \bar{P} \sigma^{\prime \prime}+\eta \psi_{\text {osc }}^{-}\left(-\sigma^{\prime \prime}\right)}: \mathrm{e}^{\frac{1}{2} \bar{Q}}
\end{aligned}
$$


where $\psi_{\mathrm{osc}}^{ \pm}$stand for the oscillator parts of $\psi^{ \pm}$. Then the quantum analogue of (3) with omission of the overall coefficient are given by

$$
\begin{aligned}
& A(\sigma)=A_{\sigma \sigma^{\prime}}+A_{\Delta}, \quad B(-\sigma)=B_{\sigma \sigma^{\prime}}+B_{\Delta}, \\
& A\left(\sigma^{\prime}\right)=A_{\sigma \sigma^{\prime}}+q^{-2 \varpi-2} A_{\Delta}, \quad B\left(-\sigma^{\prime}\right)=B_{\sigma \sigma^{\prime}}+q^{2 \varpi} B_{\Delta},
\end{aligned}
$$

where we have introduced $q \equiv \mathrm{e}^{i \pi g}$. The screening operators appearing in (11) are simply the products of these operators and, hence, can be expressed in terms of a set of four independent operators defined by

$$
x=A_{\sigma \sigma^{\prime}} B_{\sigma \sigma^{\prime}}, \quad y=A_{\sigma \sigma^{\prime}} B_{\Delta}, \quad z=A_{\Delta} B_{\sigma \sigma^{\prime}}, \quad w=A_{\Delta} B_{\Delta} .
$$

Though the operators (12) do not satisfy simple relations under interchanges in their products, (14) satisfies the exchange algebra

$$
y x=q^{2} x y, \quad z x=q^{-2} x z, \quad w x=x w=q^{-2} y z=q^{2} z y, \quad w y=q^{-2} y w, \quad w z=q^{2} z w .
$$

As for the exchange relations with the vertex operators, they commute with $V_{\lambda}(\sigma)$ and satisfy $x V_{\lambda}\left(\sigma^{\prime}\right)=V_{\lambda}\left(\sigma^{\prime}\right) x, \quad y V_{\lambda}\left(\sigma^{\prime}\right)=q^{4 \lambda} V_{\lambda}\left(\sigma^{\prime}\right) y, \quad z V_{\lambda}\left(\sigma^{\prime}\right)=q^{-4 \lambda} V_{\lambda}\left(\sigma^{\prime}\right) z, \quad w V_{\lambda}\left(\sigma^{\prime}\right)=V_{\lambda}\left(\sigma^{\prime}\right) w$

Using $[\bar{Q}, \varpi]=2$, we further find $x \varpi=(\varpi+2) x, V_{\lambda}(\sigma) \varpi=(\varpi+2 \lambda) V_{\lambda}(\sigma)$ and similar relations for $y, z, w$ and $V_{\lambda}\left(\sigma^{\prime}\right)$.

The exchange algebra (15) can be used to rearrange any product of operators (14) in a definite order. We can choose $x^{l} y^{m} w^{n}$ and $x^{l} z^{m} w^{n}(l, m, n=0,1,2, \cdots)$ as a set of independent operators. There are just $(J+1)^{2}$ independent ones for $l+m+n=J$.

We now come back to (11). By interchanging the vertex operators and the screening charges, we can move the vertex operators to the left and then divide out them from the expression. We thus obtain

$$
\begin{aligned}
\sum_{m+m^{\prime}=J} & {\left[C_{m}(\varpi-2 \nu, \lambda) C_{m^{\prime}}(\varpi+2 m, \nu)(\mathcal{S}(2 \nu))^{m}(\mathcal{S}(\varpi+1))^{m^{\prime}}\right.} \\
& \left.-C_{m}(\varpi-2 \lambda, \nu) C_{m^{\prime}}(\varpi+2 m, \lambda)(\mathcal{S}(\varpi-2 \lambda+1))^{m}(\mathcal{S}(0))^{m^{\prime}}\right]=0,
\end{aligned}
$$

where we have defined

$$
\mathcal{S}(\xi)=x+q^{2 \xi} y+q^{-2 \xi} z+w
$$


The lhs of (17) is a homogeneous polynomial in the operators (14) of order $J$. Since there appear $(J+1)^{2}$ independent operators, it yields a set of redundant $(J+1)^{2}$ recurrence relations for $C_{m}(\varpi, \lambda)$. They are redundant in the sense that only the relations associated with the operators $x^{J-1} y$ and $y^{J}$ for $\lambda=\nu$ are sufficient to determine $C_{m}(\varpi, \lambda)$. Though this approach works well for small $J$ and reproduce the results of refs. [6, 11], it is not so illuminating for arbitrary $J$.

The condition (17) can be simplified further if one notice that the exchange algebra (15) possesses a simple quantum mechanical realization [8]. By using the same notation for the quantum mechanical realization, (15) is satisfied by the operators given by

$$
x=u \mathrm{e}^{\bar{Q}}, \quad y=u^{2} q^{-\varpi-2} \mathrm{e}^{\bar{Q}}, \quad z=v q^{\varpi+2} \mathrm{e}^{\bar{Q}}, \quad w=u v \mathrm{e}^{\bar{Q}},
$$

where $u$ and $v$ are arbitrary parameters. In this realization the screening operator (18) can be written in a factorized form as

$$
\mathcal{S}(\xi)=h(-\varpi+2 \xi-2, \varpi-2 \xi+2) \mathrm{e}^{\bar{Q}}
$$

where $h(a, b)$ is given by

$$
h(a, b)=\left(1+u q^{a}\right)\left(u+v q^{b}\right) .
$$

Putting (20) into (17) and moving all the $\mathrm{e}^{\bar{Q}}$ to the right to eliminate them, we obtain

$$
\begin{gathered}
\sum_{m+m^{\prime}=J}\left\{C_{m}(\varpi-2 \nu, \lambda) C_{m^{\prime}}(\varpi+2 m, \nu) \prod_{k=1}^{m} h(-\varpi+4 \nu-2 k, \varpi-4 \nu+2 k)\right. \\
\times \prod_{l=1}^{m^{\prime}} h(\varpi+2 m+2 l-2,-\varpi-2 m-2 l+2) \\
-C_{m}\left(\varpi+2 m^{\prime}, \lambda\right) C_{m^{\prime}}(\varpi-2 \lambda, \nu) \prod_{k=1}^{m} h\left(-\varpi-2 m^{\prime}-2 k, \varpi+2 m^{\prime}+2 k\right) \\
\left.\times \prod_{l=1}^{m^{\prime}} h(\varpi-4 \lambda+2 l-2,-\varpi+4 \lambda-2 l+2)\right\}=0 .
\end{gathered}
$$

The lhs is a polynomial in $u, v$ and contains only commuting variables. There arise $(J+1)^{2}$ independent terms proportional to $u^{J+k-l} v^{l}(k, l=0,1, \cdots, J)$ in one-to-one correspondence with the $(J+1)^{2}$ independent operators appearing in (17). Since (22) holds identically for any $u$ and $v$, we can choose them arbitrarily so that the recurrence relations for $C_{m}(\varpi, \lambda)$ takes a simple form. The solution of (22) proceeds in three steps. 
The first one is to resolve the $\varpi$ dependence. We choose $u=-q^{\varpi+2 J}$ and $v=q^{4 \nu+2 J-2}$ for which $h(-\varpi-2 J, \varpi+2 J)=h(-\varpi+4 \nu-2, \varpi-4 \nu+2)=0$. In this case only the terms containing $C_{J}(\varpi, \nu)$ and $C_{J}(\varpi-2 \lambda, \nu)$ remain nonvanishing and (22) reduces to

$$
\frac{C_{J}(\varpi, \nu)}{C_{J}(\varpi-2 \lambda, \nu)}=\prod_{k=1}^{J} \frac{\sinh _{q}(\varpi-2 \lambda+J+k-1) \sinh _{q}(\varpi-2 \lambda-2 \nu+k)}{\sinh _{q}(\varpi+J+k-1) \sinh _{q}(\varpi-2 \nu+k)},
$$

where we have introduced $\sinh _{q} x \equiv \frac{q^{x}-q^{-x}}{2}$. This immediately leads to

$$
C_{m}(\varpi, \lambda)=f_{m}(\lambda) \prod_{k=1}^{m} \frac{1}{\sinh _{q}(\varpi+m+k-1) \sinh _{q}(\varpi-2 \lambda+k)} \quad(m=1,2, \cdots),
$$

where $f_{m}(\lambda)$ are yet undetermined functions of $\lambda$.

The next step is to find the $\lambda$ dependence of $f_{m}(\lambda)$. For the purpose we consider the case $u=$ $-q^{-\varpi+4 \lambda}$ and $v=q^{-2 \varpi+4 \lambda+4 \nu-2}$, where $h(\varpi-4 \lambda,-\varpi+4 \lambda)=h(-\varpi+4 \nu-2, \varpi-4 \nu+2)=0$ and only the terms containing $C_{J}(\varpi, \lambda)$ and $C_{J}(\varpi, \nu)$ remain nonvanishing in (22). This combined with (24) gives the ratio $f_{J}(\varpi, \nu)$ with $f_{J}(\varpi, \lambda)$. We thus obtain

$$
f_{m}(\lambda)=c_{m} \prod_{k=1}^{m} \sinh _{q}(2 \lambda+k-1)
$$

where $c_{m}$ is a $\lambda$ independent constant.

The last step is to find $c_{m}$. Putting $u=-q^{\varpi+2 J}$ and $v=q^{4 \nu+2 J-4}$, we get $h(-\varpi-2 J, \varpi+$ $2 J)=h(-\varpi+4 \nu-4, \varpi-4 \nu+4)=0$, for which (22) reduces to a recurrence relation between $c_{J}$ and $c_{J-1}$. This leads to

$$
c_{m}=\prod_{k=1}^{m} \frac{i a \sinh _{q} 1}{\sinh _{q} k}
$$

where $c_{1} \equiv i a$ is a yet undetermined constant.

Combining all these results, we finally obtain the expansion coefficients as

$$
C_{m}(\varpi, \lambda)=\prod_{k=1}^{m} \frac{i a \sinh _{q} 1 \sinh _{q}(2 \lambda+k-1)}{\sinh _{q} k \sinh _{q}(\varpi+m+k-1) \sinh _{q}(\varpi-2 \lambda+k)}
$$

This can be shown to coincide with the results of ref. [6] for small $J$. We see that the requirement of conformal property and locality almost completely determine the expansion (8).

Kazama and Nicolai [11] noted a solution to the locality conditions for $2 \lambda g, 2 \nu g$ and $g$ being integers. In this case the screening charges and the vertex operators appearing in (11) are mutually commuting as can be seen from (15) and (16). This implies that naive expansion of 
the classical solution holds true after the substitution $\psi \rightarrow \eta \psi$, provided the coefficients satisfy the periodicity $C_{m}\left(\varpi+g^{-1}, \lambda\right)=C_{m}(\varpi, \lambda)$. This leads to the solution of ref. [11, which cannot be obtained from (27) as a special case.

We now turn to the field equation satisfied by $\varphi$. As mentioned previously, the Liouville field can be defined by differentiating (8) with respect to $\lambda$ and then putting $\lambda=0$. This leads to

$$
\varphi=\eta \psi+\sum_{m=1}^{\infty}\left(\frac{\mu^{2}}{8}\right)^{m} \frac{2 \pi i g\left(i a \sinh _{q} 1\right)^{m}}{\sinh _{q} m \sinh _{q}(\varpi+m)} \prod_{k=1}^{2 m-1} \frac{1}{\sinh _{q}(\varpi+k)} S^{m},
$$

where use has been made of the relations

$$
C_{m}(\varpi, 0)=\delta_{m, 0},\left.\quad \frac{d}{d \lambda} C_{m}(\varpi, \lambda)\right|_{\lambda=0}=\frac{2 \pi i g\left(i a \sinh _{q} 1\right)^{m}}{\sinh _{q} m \sinh _{q}(\varpi+m)} \prod_{k=1}^{2 m-1} \frac{1}{\sinh _{q}(\varpi+k)} .
$$

Noting that the screening operator satisfies

$$
\begin{aligned}
& \partial_{ \pm} S^{m}=\frac{\sinh _{q} m \sinh _{q}(\varpi+m)}{\sinh _{q} 1 \sinh _{q}(\varpi+1)} \partial_{ \pm} S S^{m-1} \\
& \partial_{+} \partial_{-} S^{m}=\left(\frac{2 \sinh _{q} m \sinh _{q}(\varpi+m)}{\sinh _{q} 1}\right)^{2} V_{1} S^{m-1}
\end{aligned}
$$

we obtain

$$
\begin{aligned}
\partial_{+} \partial_{-} \varphi=-\pi g a \mu^{2} V_{1} \sum_{m=0}^{\infty} & \left(\frac{\mu^{2}}{8}\right)^{m}\left(i a \sinh _{q} 1\right)^{m} \frac{\sinh _{q}(m+1) \sinh _{q}(\varpi+m-1)}{\sinh _{q} 1} \\
& \times \prod_{k=1}^{2 m+1} \frac{1}{\sinh _{q}(\varpi+k-2)} S^{m} .
\end{aligned}
$$

Since the rhs just coincides to (8) multiplied by $-\pi g a \mu^{2}$ for $\lambda=1$, the Liouville equation is satisfied for the choice

$$
4 \pi g a=1
$$

This determines the unknown constant $a$.

What remains to show is the canonical commutation relations. This can be done along the strategy for the proof of locality. We first assume the expansion

$$
\varphi(\tau, \sigma)=\eta \psi(\tau, \sigma)+2 \pi i g \sum_{m=1}^{\infty}\left(\frac{\mu^{2}}{2}\right)^{m} D_{m}(\varpi)(\bar{S}(\tau, \sigma))^{m}
$$

and then determine $D_{m}(\varpi)$ so as for the Liouville field to satisfy the commutation relations

$$
\left[\varphi(0, \sigma), \partial_{+} \varphi\left(0, \sigma^{\prime}\right)\right]=\left[\eta \psi(0, \sigma), \eta \partial_{+} \psi\left(0, \sigma^{\prime}\right)\right]
$$


where we have introduced the notation $\bar{S}=\left(2 \sinh _{q}(\varpi+1)\right)^{-2} S$. Using (30), we can equivalently write (34) as

$$
\begin{aligned}
& {\left[\eta \psi(0, \sigma), \frac{\sinh _{q} J \sinh _{q}(\varpi+J)}{\sinh _{q} 1 \sinh _{q}(\varpi+1)} D_{J}(\varpi) \partial_{+} \bar{S}\left(0, \sigma^{\prime}\right) \bar{S}^{J-1}\left(0, \sigma^{\prime}\right)\right]+D_{J}(\varpi)\left[\bar{S}^{J}(0, \sigma), \eta \partial_{+} \psi\left(0, \sigma^{\prime}\right)\right]} \\
& +2 \pi i g \sum_{m+m^{\prime}=J}\left[D_{m}(\varpi) \bar{S}^{m}(0, \sigma), \frac{\sinh _{q} m^{\prime} \sinh _{q}\left(\varpi+m^{\prime}\right)}{\sinh _{q} 1 \sinh _{q}(\varpi+1)} D_{m^{\prime}}(\varpi) \partial_{+} \bar{S}\left(0, \sigma^{\prime}\right) \bar{S}^{m^{\prime}-1}\left(0, \sigma^{\prime}\right)\right] \\
& \quad=0
\end{aligned}
$$

To simplify the lhs we need some commutation relations. For $0<\sigma^{\prime}<\sigma<2 \pi$ we get

$$
\begin{aligned}
& {\left[\bar{S}(0, \sigma), \eta \partial_{+} \psi\left(0, \sigma^{\prime}\right)\right]=-\left[\eta \psi(0, \sigma), \partial_{+} \bar{S}\left(0, \sigma^{\prime}\right)\right]=\frac{2 \pi i g}{\sinh _{q}(\varpi+1)} q^{\varpi+1} \partial_{+} \bar{A}\left(\sigma^{\prime}\right) \bar{B}(-\sigma)} \\
& {\left[\eta \psi(0, \sigma), \bar{S}\left(0, \sigma^{\prime}\right)\right]=-\frac{2 \pi i g}{\sinh _{q}(\varpi+1)}\left\{q^{\varpi+1} \bar{A}\left(\sigma^{\prime}\right) \bar{B}(-\sigma)+q^{-\varpi-1} \bar{A}(\sigma) \bar{B}\left(-\sigma^{\prime}\right)\right\}}
\end{aligned}
$$

where we have introduced rescaled fields $\bar{A}=A\left(2 \sinh _{q} \varpi\right)^{-1}$ and $\bar{B}=\left(2 \sinh _{q} \varpi\right)^{-1} B$. These are the quantum analogue of (3) The extra factor $\left(2 \sinh _{q} \varpi\right)^{-1}$ corresponds to the coefficient $C(P)$ of the classical formula (3). The commutation relations (36) imply that the lhs of (35) is linear in $\partial_{+} A\left(\sigma^{\prime}\right)$, which can be moved to the left by using the relation

$$
\bar{S}(0, \sigma) \partial_{+} A\left(\sigma^{\prime}\right)=\frac{\sinh _{q}(\varpi+3)}{\sinh _{q}(\varpi+1)} \partial_{+} A\left(\sigma^{\prime}\right) B(-\sigma)\left(A_{\sigma \sigma^{\prime}}+q^{-4} A_{\Delta}\right) .
$$

We then replace $\partial_{+} A\left(\sigma^{\prime}\right)$ with $A\left(\sigma^{\prime}\right)$ in the resulting formula. After these manipulations (35) can be converted into the form similar to (17), to which we can apply the quantum mechanical realization (19). Using the argument leading from (17) to (22), we obtain

$$
\begin{aligned}
& \left\{i \pi g \frac{\partial}{\partial \varpi} \ln \left(D_{J}(\varpi) \frac{\sinh _{q}(\varpi+J)}{\sinh _{q}(\varpi+1)}\right) D_{J}(\varpi)\right. \\
& -D_{J}(\varpi) \sum_{k=0}^{J-1} \frac{q^{\varpi+2 k+1}}{\sinh _{q}(\varpi+2 k+2)} \frac{h(-\varpi-2 k-2,-\varpi-2 k)}{h(\varpi+2 k,-\varpi-2 k)} \\
& \left.-D_{J}(\varpi) \sum_{k=1}^{J-1} \frac{q^{-\varpi-2 k-1}}{\sinh _{q}(\varpi+2 k+2)} \frac{h(\varpi+2 k, \varpi+2 k+2)}{h(\varpi+2 k,-\varpi-2 k)}\right\} \\
& \quad \times \prod_{l=1}^{J} h(\varpi+2 l-2,-\varpi-2 l+2) \\
& +\frac{D_{J}(\varpi) \sinh _{q} 1}{\sinh _{q} J \sinh _{q}(\varpi+J)} \sum_{m+m^{\prime}=J} q^{\varpi+2 m+1} h(-\varpi-2,-\varpi) \\
& \quad \times \prod_{k=1}^{m-1} h(-\varpi-2 k-2, \varpi-2 k+2) \prod_{l=1}^{m^{\prime}} h(-\varpi-2 m-2 l, \varpi+2 m+2 l)
\end{aligned}
$$




$$
\begin{aligned}
& +\sum_{m+m^{\prime}=J} \frac{\sinh _{q} m^{\prime} \sinh _{q}(\varpi+J+m)}{\sinh _{q} J \sinh _{q}(\varpi+J)} D_{m}(\varpi) D_{m^{\prime}}(\varpi+2 m) \\
& \quad \times h(-\varpi-2,-\varpi) h(\varpi+2 m, \varpi+2 m-2) \\
& \quad \times \prod_{k=1}^{m-1} h(-\varpi-2 k-2, \varpi+2 k-2) \prod_{l=1}^{m^{\prime}-1} h(\varpi+2 m+2 l,-\varpi-2 m-2 l) \\
& -\sum_{m+m^{\prime}=J} \frac{\sinh _{q} m \sinh _{q}(\varpi+m)}{\sinh _{q} J \sinh _{q}(\varpi+J)} D_{m}(\varpi) D_{m^{\prime}}(\varpi+2 m) \\
& \quad \times \prod_{k=1}^{m} h(\varpi+2 k-2,-\varpi-2 k+2) \prod_{l=1}^{m^{\prime}} h(-\varpi-2 m-2 l, \varpi+2 m+2 l)=0 .
\end{aligned}
$$

The recurrence relation for $D_{m}(\varpi)$ can be solved in the manner similar to (22). We find

$$
D_{m}(\varpi)=\frac{\left(i a \sinh _{q} 1\right)^{m} \sinh _{q}(\varpi+2 m)}{\sinh _{q} m \sinh _{q}(\varpi+m)} \prod_{k=1}^{m} \frac{\sinh _{q}(\varpi+2 k-1)}{\sinh _{q}(\varpi+2 k)} .
$$

This coincides with the coefficients obtained from (28), establishing the commutation relation (34). The transformation (28) thus induces a operatorial canonical transformation.

There remain some points to be clarified in our argument. For instance we should verify that the condition (22) is satisfied for (27) for arbitrary $u$ and $v$. Related to this is the origin of the consistency of the redundant conditions for the expansion coefficients mentioned previousely. We will discuss these issues elsewhere.

In conclusion it is possible to deform the classical Liouville field to satisfy the operator field equation and the canonical commutation relations by utilizing the degree of zero mode momentum. The formal expansion of the Liouville exponential given ref. [6] is correct to all order in the cosmological constant and for arbitrary parameters $\lambda$ and $g$. 


\section{References}

[1] J. -L Gervais and A. Neveu, Nucl. Phys. B199 (1982) 59; B209 (1982) 125; B224 (1983) 329; B238 (1984) 125.

[2] T. L. Curtright and C. B. Thorn, Phys. Rev. Lett. 48 (1982) 1309;

E. Braaten, T. Curtright, G. Ghandour and C. Thorn, Phys. Rev. Lett. 51 (1983) 19;

T. Yoneya, Phys. Lett. B148 (1984) 111;

L. Johansson, A.Kihlberg and R. Marnelius, Phys. Rev. D29 (1984) 2798;

L. Johansson and R. Marnelius, Nucl. Phys. B254 (1985) 201.

[3] E. D'Hoker and R. Jackiw, Phys. Rev. D26 (1982) 3517;

E. D'Hoker, D. Z. Freedman and R. Jackiw, Phys. Rev. D28 (1983) 2583.

[4] E. Braaten, T. Curtright and C. Thorn, Ann. Phys. (N.Y.) 147 (1983) 365;

[5] I. A. Fedseev and A. N. Leznov, Phys. Lett. B141 (1984) 100

[6] H. J. Otto and G. Weigt, Phys. Lett. B159 (1985) 341; Z. Phys. C31 (1986) 219.

[7] O. Babelon, Phys. Lett. B215 (1988) 523;

J. -L. Gervais, Commun. Math. Phys. 130 (1990) 257; 138 (1991) 301.

[8] J. -L. Gervais and J. Schnittger, Phys. Lett. B315 (1993) 258;

J. -L. Gervais and J. Schnittger, Nucl. Phys. bf B431 (1994) 273;

J. Schnittger, Theor. Math. Phys. 104 (1996) 892 and references cited therein.

[9] G. Weigt, talk given at 1992 John Hopkins Workshop on Current Problems in Particle Theory, Goteborg, Sweden, hep-th/908075, print-92-0383 (DESY-IFH).

[10] J. -L. Gervais and J. Schnittger, Nucl. Phys. B413 (1994) 433.

[11] Y. Kazama and H. Nicolai, Int. J, Mod. Phys. A9 (1994) 667.

[12] O. Babelon, Commun. Math. Phys. 139 (1991) 619. 